Rapid Reviews COVID-19

\title{
Review 2: "Molecular Docking Studies of Aromatherapy Oils Against SARS-COV-2"
}

\section{Umakanta Tripathy ${ }^{1}$, Janmejaya Rout ${ }^{2}$, Anand Kant Das ${ }^{3}$}

IIndian Institute of Technology (Indian School of Mines): Indian Institute of Technology, Physics, India,

${ }^{2}$ Indian Institute of Technology (Indian School of Mines) Dhanbad, India,

${ }^{3}$ New York University, Abu Dhabi

Published on: Nov 22, 2020

DOI: $10.1162 / 2 \mathrm{e} 3983 f 5.02 \mathrm{~d} 2584 \mathrm{~d}$

License: Creative Commons Attribution 4.0 International License (CC-BY 4.0). 


\section{$\underline{\text { RR:C19 Evidence Scale rating by reviewer: }}$}

- Potentially informative. The main claims made are not strongly justified by the methods and data, but may yield some insight. The results and conclusions of the study may resemble those from the hypothetical ideal study, but there is substantial room for doubt. Decision-makers should consider this evidence only with a thorough understanding of its weaknesses, alongside other evidence and theory. Decisionmakers should not consider this actionable, unless the weaknesses are clearly understood and there is other theory and evidence to further support it.

******************************************

\section{Review:}

The authors have carried out docking and absorption, distribution, metabolism, and excretion (ADME)/toxicity analysis of seven compounds found in several aromatic oils with SARS-COV-2 spike protein, human ACE2, and TMPRSS2 proteins. Based on their results, the authors propose that some of these compounds should potentially be explored for their therapeutic efficacy against SARS-COV-2 infection. The manuscript is well-written. However, it may be premature for publication in a journal due to few inadequacies as below:

(1) The major drawback of this study is the lack of Molecular Dynamics (MD) simulation to assess the flexibility and stability of the proposed interactions. Generally, during screening, molecular docking studies are performed to gain insights into the binding mode and crucial molecular interactions of the selected ligands. However, the stability of interaction will majorly determine if these interactions are indeed relevant and warrant further investigation. Therefore, it is crucial to carry our MD simulation studies with promising candidates.

(2) The authors have used Camostat mesylate as a standard for their analysis. Almost all the molecules studied have affinities that are neither equal to nor greater than the standard. When molecules have affinities lower than that of the standard, then the physiological relevance of the interaction becomes doubtful.

(3) The authors should make the methodology section more descriptive and include information about the search space dimension, center coordinates, exhaustiveness, etc. 
(4) Another criticism is the conclusion drawn by the authors that trans-pinocarveol has the least binding energy among the EO compounds, which is not true, as seen in Table 1 . 